MATHEMATICS OF COMPUTATION

Volume 71, Number 238, Pages 583-604

S 0025-5718(01)01339-4

Article electronically published on October 25, 2001

\title{
A STOCHASTIC PARTICLE NUMERICAL METHOD FOR 3D BOLTZMANN EQUATIONS WITHOUT CUTOFF
}

\author{
NICOLAS FOURNIER AND SYLVIE MÉLÉARD
}

\begin{abstract}
Using the main ideas of Tanaka, the measure-solution $\left\{P_{t}\right\}_{t}$ of a 3-dimensional spatially homogeneous Boltzmann equation of Maxwellian molecules without cutoff is related to a Poisson-driven stochastic differential equation. Using this tool, the convergence to $\left\{P_{t}\right\}_{t}$ of solutions $\left\{P_{t}^{l}\right\}_{t}$ of approximating Boltzmann equations with cutoff is proved. Then, a result of Graham-Méléard is used and allows us to approximate $\left\{P_{t}^{l}\right\}_{t}$ with the empirical measure $\left\{\mu_{t}^{l, n}\right\}_{t}$ of an easily simulable interacting particle system. Precise rates of convergence are given. A numerical study lies at the end of the paper.
\end{abstract}

\section{INTRODUCTION}

Our aim in this paper is to present a probabilistic interpretation of some Boltzmann equations and to use this interpretation to construct some approximating stochastic particle systems. Then we will deduce from this construction a simple algorithm for the simulation of the solutions of these equations. In this setting, deterministic particle methods seem difficult to develop, whereas this stochastic particle method is very natural in a probabilistic point of view and very simple to implement.

We will consider here spatially homogeneous Boltzmann equations of Maxwellian molecules without cutoff in $\mathbb{R}^{3}$. The main idea is to associate with such a Boltzmann equation a stochastic pure jump process of which the time-marginals flow is a measure-valued solution of the equation. Because of the possible explosions of the jump measure which is just assumed to have a second order moment, this process will be defined as a solution of a nonlinear stochastic differential equation driven by a compensated Poisson measure, and we will work in $L^{2}$-spaces. The nonlinearity is modeled by adding an auxiliary random space.

This paper is a generalization in dimension 3 of Desvillettes-Graham-Méléard 4] concerning a Kac equation without cutoff and of Fournier 5] for the dimension 2. Both were inspired by an original idea of representation due to Tanaka [16. In [16], the jump measure has a one-order moment and one can directly use Poisson point processes.

In dimension 3 , difficulties appear due to the parametrization of a sphere in $\mathbb{R}^{3}$. We are not in a good Lipschitz context. However, we prove a sort of Lipschitz property for the amplitude of the jumps, which is sufficient to define properly

Received by the editor March 14, 2000.

2000 Mathematics Subject Classification. Primary 60J75, 60H10, 60K35, 82C40.

Key words and phrases. Boltzmann equations without cutoff, stochastic differential equations, jump measures, interacting particle systems. 
the solutions of the nonlinear stochastic differential equation and to deduce the existence of a measure-valued solution for the associated Boltzmann equation. But nevertheless, we are not able, for the moment, to obtain by this approach the existence and regularity of a function-valued solution, as in dimension 1 or 2 .

Next we approximate the law of the stochastic process by simulable interacting particle systems, proving a generalized law of large numbers on a path space. We use a result of Graham-Méléard [6] who discuss this problem in a general context, but in a cutoff case. Thus we consider first cutoff approximations of our model and associate with each cutoff model some cutoff approximating interacting particle systems. We prove the convergence of the cutoff model to the model without cutoff. We obtain precise rates of convergence, which are easily computable. We conclude by choosing a sequence of cutoff approximations indexed by the size $n$ of the approximating particle systems and converging to 0 as $n$ tends to infinity in good asymptotics such that finally the empirical measures of the particle systems converge to the solution of the Boltzmann equation without cutoff.

It is then easy to describe some simulation algorithms. We finally give some numerical results at the end of the paper.

Horowitz and Karandikar, in [8, develop a similar approach in a theoretical point of view. The particle systems they consider are not simulable and they don't obtain rates of convergence.

Let us also mention that Sznitman, in [14], studies a spatially homogeneous hardsphere Boltzmann equation taking into account the large velocities, but without angular dependence in the collision kernel. He obtains convergence results without estimates using a compactness-uniqueness method.

Notation. $K$ will denote a constant which may change from line to line.

$|v|$ or $|X|$ will denote the euclidian norm in $\mathbb{R}^{3}$ of the vectors $v$ or $X$.

For a Polish space $E, \mathcal{P}(E)$ will denote the space of probability measures on $E$. It will be endowed with the topology of the weak convergence. $\mathcal{P}_{2}(E)$ will be the subspace of probability measures with a second order moment.

If $V$ is a random variable on a probability space, $\mathcal{L}(V)$ will denote the law of $V$.

The Skorohod space $\mathbb{D}\left(\mathbb{R}_{+}, \mathbb{R}^{3}\right)$ is the set of all right-continuous and left-handed limited functions from $\mathbb{R}_{+}$to $\mathbb{R}^{3}$.

\section{The 3D Boltzmann EQUATion}

We consider here a spatially homogeneous Boltzmann equation

$$
\frac{\partial f}{\partial t}=Q(f, f)
$$

where $Q$ is a quadratic collision kernel acting on the variable $v$, preserving momentum and kinetic energy, of the form

$$
\begin{array}{r}
Q(f, f)(t, v)=\int_{v_{*} \in \mathbb{R}^{3}} \int_{\varphi=0}^{2 \pi} \int_{\theta=0}^{\pi}\left(f\left(t, v^{\prime}\right) f\left(t, v_{*}^{\prime}\right)-f(t, v) f\left(t, v_{*}\right)\right) \\
\beta\left(\left|v-v_{*}\right|, \theta\right) \sin \theta d \theta d \varphi d v_{*}
\end{array}
$$

with $v^{\prime}=\frac{v+v_{*}}{2}+\frac{\left|v-v_{*}\right|}{2} \sigma$ and $v_{*}^{\prime}=\frac{v+v_{*}}{2}-\frac{\left|v-v_{*}\right|}{2} \sigma$, the unit vector $\sigma$ having colatitude $\theta$ and longitude $\varphi$ in the spherical coordinates in which $v-v_{*}$ is the polar axis. The nonnegative function $\beta$ is called the cross section. 
If the molecules in the gas interact according to an inverse power law in $1 / r^{s}$ with $s \geq 2$, then $\beta(z, \theta)=z^{\frac{s-5}{s-1}} d(\theta)$ where $\left.\left.d \in L_{\mathrm{loc}}^{\infty}(] 0, \pi\right]\right)$ and $d(\theta) \sin \theta \sim K(s) \theta^{-\frac{s+1}{s-1}}$ when $\theta$ goes to zero, for some $K(s)>0$. Physically, this explosion comes from the accumulation of grazing collisions. This equation seems very difficult to study and we will restrict our attention to the case of Maxwellian molecules for which the cross section $\beta(z, \theta) \sin \theta=\beta(\theta)$ depends only on $\theta$. The only condition we assume, following the physical behaviour, is that

$$
\int_{0}^{\pi} \theta^{2} \beta(\theta) d \theta<+\infty
$$

Equation (2.1) has to be understood in a weak sense. By a standard integration by parts, we define a solution $f$ as satisfying for each $\phi \in C_{b}^{2}\left(\mathbb{R}^{3}\right)$

$$
\frac{\partial}{\partial t} \int_{\mathbb{R}^{3}} f(t, v) \phi(v) d v=\int_{\mathbb{R}^{3} \times \mathbb{R}^{3}} \int_{0}^{2 \pi} \int_{0}^{\pi}\left(\phi\left(v^{\prime}\right)-\phi(v)\right) \beta(\theta) d \theta d \varphi f(t, v) d v f\left(t, v_{*}\right) d v_{*} .
$$

But here the right-hand side term may explode, since the function $\beta$ may have an infinite mass on $[0, \pi]$. Thus it has to be compensated and the definition of the solutions of (2.1) is as follows :

Definition 2.1. We say that a probability measure flow $\left(P_{t}\right)_{t}$ is a measure-solution of the Boltzmann equation (2.1) if for each $\phi \in C_{b}^{2}\left(\mathbb{R}^{3}\right)$

$$
\left\langle\phi, P_{t}\right\rangle=\left\langle\phi, P_{0}\right\rangle+\int_{0}^{t}\left\langle K_{\beta}^{\phi}\left(v, v^{*}\right), P_{s}(d v) P_{s}\left(d v^{*}\right)\right\rangle d s
$$

where $K_{\beta}^{\phi}$ is defined in the compensated form

$$
\begin{aligned}
K_{\beta}^{\phi}(v, v *)= & -b\left(v-v_{*}\right) \cdot \nabla \phi(v) \\
& +\int_{0}^{2 \pi} \int_{0}^{\pi}\left(\phi\left(\frac{v+v_{*}}{2}+\frac{\left|v-v_{*}\right|}{2} \sigma\right)-\phi(v)\right. \\
& \left.-\left(\frac{v_{*}-v}{2}+\frac{\left|v-v_{*}\right|}{2} \sigma\right) \cdot \nabla \phi(v)\right) \beta(\theta) d \theta d \varphi
\end{aligned}
$$

with

$$
b=\pi \int_{0}^{\pi}(1-\cos \theta) \beta(\theta) d \theta .
$$

Indeed, we can remark that the unit vector $\sigma$ writes

$$
\sigma=\cos \theta \frac{v-v_{*}}{\left|v-v_{*}\right|}+\sin \theta \frac{\Gamma\left(v-v_{*}, \varphi\right)}{\left|v-v_{*}\right|}
$$

where for $X \in \mathbb{R}^{3}, \varphi \in[0,2 \pi[$,

$$
\Gamma(X, \varphi)=\cos \varphi I(X)+\sin \varphi J(X)
$$


and $\left(\frac{X}{|X|}, \frac{I(X)}{|X|} \frac{J(X)}{|X|}\right)$ is an orthonormal basis of $\mathbb{R}^{3}$. One can choose, for example,

$$
\begin{aligned}
& I(X)= \begin{cases}\frac{|X|}{\sqrt{X_{x}^{2}+X_{y}^{2}}}\left(-X_{y}, X_{x}, 0\right) & \text { if } X_{x}^{2}+X_{y}^{2}>0 \\
\left(X_{z}, 0,0\right) & \text { else }\end{cases} \\
& J(X)=\frac{X}{|X|} \wedge I(X) .
\end{aligned}
$$

Then

$$
v^{\prime}=\frac{v+v_{*}}{2}+\cos \theta \frac{v-v_{*}}{2}+\frac{1}{2} \sin \theta \Gamma\left(v-v_{*}, \varphi\right)
$$

and if we set

$$
a\left(v, v_{*}, \theta, \varphi\right)=v^{\prime}-v=\frac{\cos \theta-1}{2}\left(v-v_{*}\right)+\frac{\sin \theta}{2} \Gamma\left(v-v_{*}, \varphi\right),
$$

we observe that the first term of the right-hand side term of $a\left(v, v_{*}, \theta, \varphi\right)$ is integrable with respect to $\theta$ and that the second term is odd and nonintegrable in $\theta$, but its square is integrable. Then

$$
\begin{aligned}
& K_{\beta}^{\phi}(v, v *)=-b\left(v-v_{*}\right) \cdot \nabla \phi(v) \\
& \quad+\int_{0}^{2 \pi} \int_{0}^{\pi}\left(\phi\left(v+a\left(v, v_{*}, \theta, \varphi\right)\right)-\phi(v)-a\left(v, v_{*}, \theta, \varphi\right) \cdot \nabla \phi(v)\right) \beta(\theta) d \theta d \varphi
\end{aligned}
$$

The following result is due to Toscani-Villani, 17, Theorem 5.

Theorem 2.2. Assume that $P_{0}$ is a probability measure on $\mathbb{R}^{3}$ admitting a moment of order 2 and that $\beta$ is a cross section satisfying $\int_{0}^{\pi} \theta^{2} \beta(\theta) d \theta<\infty$. The uniqueness of a measure-solution holds for the Boltzmann equation (2.3).

We thus deduce the following important remark.

Remark 2.3. Thanks to Theorem 2.2, we are sure that in the case where the existence of a weak function-solution $f(t, v)$ to (2.1) holds for $P_{0} \in \mathcal{P}_{2}\left(\mathbb{R}^{3}\right)$, the measure-solution $P_{t}$ we will study in the sequel is given by $P_{t}(d v)=f(t, v) d v$.

We have conservation of mass in (2.3), which leads to a probabilistic approach. That consists in considering (2.3) as the evolution equation of the flow of marginals of a Markov process of which the law is defined by a martingale problem.

Definition 2.4. Let $\beta$ be a cross section such that $\int_{0}^{\pi} \theta^{2} \beta(\theta) d \theta<+\infty$ and $P_{0}$ in $\mathcal{P}_{2}\left(\mathbb{R}^{3}\right)$. We say that $P \in \mathcal{P}\left(\mathbb{D}\left(\mathbb{R}+, \mathbb{R}^{3}\right)\right)$ solves the nonlinear martingale problem $\operatorname{MP}\left(\beta, P_{0}\right)$ starting at $P_{0}$ if for $V$ the canonical process, the law of $V_{0}$ under $P$ is $P_{0}$ and for any $\phi \in C_{b}^{2}\left(\mathbb{R}^{3}\right)$,

$$
\phi\left(V_{t}\right)-\phi\left(V_{0}\right)-\int_{0}^{t}\left\langle K_{\beta}^{\phi}\left(V_{s}, v_{*}\right), P_{s}\left(d v_{*}\right)\right\rangle d s
$$

is a square-integrable $P$-martingale. Here, the nonlinearity appears through $P_{s}$ which denotes the marginal of $P$ at time $s$. 
Remark 2.5. If $P$ is a solution of $\operatorname{MP}\left(\beta, P_{0}\right)$, then its marginal flow $\left\{P_{t}\right\}_{t}$ is a measure-solution of the associated Boltzmann equation, in the sense of Definition (2.1).

We generalize here the results of Tanaka (in which $\beta$ integrates $\theta$ ) to the case where $\beta$ integrates only $\theta^{2}$. In a probabilistic point of view, that means that instead of working with some Poisson point measures, we use their compensated measures. The main difference with a similar approach for the Boltzmann equation in dimensions one or two (cf. 4, [5]) comes from the spherical parameter $\varphi$. The velocity $v^{\prime}$ after collision is a function of $v, v_{*}, \theta$ and $\varphi$. As a function of $\varphi$, it is periodic on $\mathbb{R}$ with period $2 \pi$. This function depends on the choice of the origin $\varphi=0$ in a spherical coordinate system on the sphere $S_{v, v_{*}}$ with center $\frac{v+v_{*}}{2}$ and diameter $\left|v-v_{*}\right|$. We cannot find a choice of the origin $\varphi=0$ implying the smoothness of $a\left(v, v_{*}, \theta, \varphi\right)$ in the variables $v$ and $v_{*}$. Nevertheless, we begin by proving a "fine" version of a result due to Tanaka, [16], Lemma 3.1, which gives a sort of Lipschitz property for $a$, sufficient for our study.

Lemma 2.6. 1 . There exists a measurable function $\varphi_{0}: \mathbb{R}^{3} \times \mathbb{R}^{3} \mapsto[0,2 \pi[$, such that for all $X, Y$, all $\varphi$,

$$
\left|\Gamma(X, \varphi)-\Gamma\left(Y, \varphi+\varphi_{0}(X, Y)\right)\right| \leq 3|X-Y|
$$

(all the angles are modulo $2 \pi$ ).

2. This implies that for all $v, v_{*}, w, w_{*}$ in $\mathbb{R}^{3}, \theta \in[0, \pi], \varphi \in[0,2 \pi]$,

$$
\begin{array}{r}
\left|a\left(v, v_{*}, \theta, \varphi\right)-a\left(w, w_{*}, \theta, \varphi+\varphi_{0}\left(v-v_{*}, w-w_{*}\right)\right)\right| \\
\leq 2 \theta\left(|v-w|+\left|v_{*}-w_{*}\right|\right)
\end{array}
$$

and in particular that for all $v, v_{*}, \theta, \varphi$,

$$
\left|a\left(v, v_{*}, \theta, \varphi\right)\right| \leq 2 \theta\left(|v|+\left|v_{*}\right|\right) .
$$

Proof. We just have to check 1., 2. being then an immediate consequence. For any $X \in \mathbb{R}^{3}$, we set

$$
C_{X}=\left\{U \in \mathbb{R}^{3} /|U|=|X| \text { and }\langle U, X\rangle=0\right\}
$$

Notice that for any $X \in \mathbb{R}^{3}, C_{X}=\{\Gamma(X, \varphi) / \varphi \in[0,2 \pi[\}$. Then we fix $X$ and $Y$ in $\mathbb{R}^{3}$. First notice that if $X$ and $Y$ are colinear, it suffices to choose $\varphi_{0}(X, Y)=0$. We thus now assume that $X$ and $Y$ are not colinear. Then we build some transformations of $\mathbb{R}^{3}$. First, we set, for $U \in \mathbb{R}^{3}, h(U)=\frac{|Y|}{|X|} U$.

We also denote by $\rho$ the rotation of $\mathbb{R}^{3}$ tranforming $\frac{X}{|X|}$ into $\frac{Y}{|Y|}$ around a line perpendicular to the plane determined by $X$ and $Y$. The linear map $\tau=$ $\rho \circ h: \mathbb{R}^{3} \mapsto \mathbb{R}^{3}$ transforms $C_{X}$ into $C_{Y}$. Since $\Gamma(X, 0)$ belongs to $C_{X}$, we deduce that $\tau(\Gamma(X, 0))$ belongs to $C_{Y}$, thus there exists $\varphi_{0}(X, Y) \in[0,2 \pi[$ such that $\tau(\Gamma(X, 0))=\Gamma\left(Y, \varphi_{0}(X, Y)\right)$ and it is not hard to deduce that for all $\varphi$,

$$
\tau(\Gamma(X, \varphi))=\Gamma\left(Y, \varphi+\varphi_{0}(X, Y)\right) .
$$


Thus

$$
\begin{aligned}
\left|\Gamma(X, \varphi)-\Gamma\left(Y, \varphi+\varphi_{0}(X, Y)\right)\right|=|\Gamma(X, \varphi)-\tau(\Gamma(X, \varphi))| \\
\quad \leq\left|\Gamma(X, \varphi)-\frac{|Y|}{|X|} \Gamma(X, \varphi)\right|+\left|\frac{|Y|}{|X|} \Gamma(X, \varphi)-\frac{|Y|}{|X|} \rho(\Gamma(X, \varphi))\right| \\
\quad \leq\left(1-\frac{|Y|}{|X|}\right)|X|+\frac{|Y|}{|X|}|X| \times\|\operatorname{Id}-\rho\|_{\text {op }} \\
\quad \leq|X-Y|+|Y|\left|\frac{X}{|X|}-\frac{Y}{|Y|}\right| \leq 3|X-Y| .
\end{aligned}
$$

\section{The CASE With CUtofF}

We first consider in this section the simpler so-called cutoff case for which $\int_{0}^{\pi} \beta(\theta) d \theta<+\infty$. Existence and uniqueness of a solution $P^{\beta}$ of the nonlinear martingale problem $\operatorname{MP}\left(\beta, P_{0}\right)$ will be easily proved. In the case where there exists a unique solution $f^{\beta}$ to the Boltzmann equation, the law $P_{t}^{\beta}$ has for each $t$ the density $f_{t}^{\beta}$. Moreover, we will describe some simulable interacting particle systems of which the law converges to $P^{\beta}$ when the size of the system tends to infinity. This section is standard and is almost contained in [4] which concerns the onedimensional case. But it is necessary to develop it for a good understanding of the case without cutoff.

Theorem 3.1. Let $\beta$ be a cross section such that $\|\beta\|_{1}=\int_{0}^{\pi} \beta(\theta) d \theta<+\infty$ and $P_{0} \in \mathcal{P}(\mathbb{R})$. There exists a unique solution $P^{\beta}$ to the nonlinear martingale problem $\operatorname{MP}\left(\beta, P_{0}\right)$. Its flow of time-marginals $\left(P_{t}^{\beta}\right)_{t \geq 0}$ is the unique (probability measure flow) solution of the equation (2.3).

Proof. We follow Shiga-Tanaka, [13], Lemma 2.3, and more recently DesvillettesGraham-Méléard, 4. Since $\beta$ is in $L^{1}([0, \pi])$ and is even, the jump operator has the simpler form: $\forall \phi \in C_{b}^{2}\left(\mathbb{R}^{3}\right)$,

$$
K_{\beta}^{\phi}(v, v *)=\int_{0}^{2 \pi} \int_{0}^{\pi}\left(\phi\left(\frac{v+v_{*}}{2}+\frac{\left|v-v_{*}\right|}{2} \sigma\right)-\phi(v)\right) \beta(\theta) d \theta d \varphi
$$

and moreover for any flow $\left(Q_{t}\right)_{t}$ in $\mathbb{D}\left(\mathbb{R}+, \mathcal{P}\left(\mathbb{R}^{3}\right)\right)$

$$
\phi \in L^{\infty}\left(\mathbb{R}^{3}\right) \Rightarrow\left\langle K_{\beta}^{\phi}(., v *), Q_{s}\left(d v_{*}\right)\right\rangle \in L^{\infty}\left(\mathbb{R}^{3}\right) .
$$

Then the operator $\phi \mapsto\left\langle K_{\beta}^{\phi}(., v *), Q_{s}\left(d v_{*}\right)\right\rangle$ is a pure-jump Markov operator generating a unique law $P^{Q}$ in $\mathcal{P}\left(\mathbb{D}\left(\mathbb{R}+, \mathbb{R}^{3}\right)\right)$ starting at $P_{0}$. Its flow of marginals satisfies a linear evolution equation: for $\phi \in L^{\infty}\left(\mathbb{R}^{3}\right)$,

$$
\left\langle\phi, P_{t}^{Q}\right\rangle=\left\langle\phi, P_{0}\right\rangle+\int_{0}^{t}\left\langle K_{\beta}^{\phi}\left(v, v^{*}\right), P_{s}^{Q}(d v) Q_{s}\left(d v^{*}\right)\right\rangle d s .
$$

Let $|\mu|=\sup \left\{\langle\phi, \mu\rangle:\|\phi\|_{\infty} \leq 1\right\}$ denote the variation norm. For $\left(Q_{t}^{i}\right)_{t \geq 0}, i=1,2$, and the corresponding solutions $\left(P_{t}^{Q^{i}}\right)_{t \geq 0}$,

$$
\left\langle\phi, P_{t}^{Q^{1}}-P_{t}^{Q^{2}}\right\rangle=\int_{0}^{t}\left\langle K_{\beta}^{\phi}, Q_{s}^{1} \otimes\left(P_{s}^{Q^{1}}-P_{s}^{Q^{2}}\right)+\left(Q_{s}^{1}-Q_{s}^{2}\right) \otimes P_{s}^{Q^{2}}\right\rangle d s
$$


and hence

$$
\left|P_{t}^{Q^{1}}-P_{t}^{Q^{2}}\right| \leq 4 \pi\|\beta\|_{1} \int_{0}^{t}\left(\left|P_{s}^{Q^{1}}-P_{s}^{Q^{2}}\right|+\left|Q_{s}^{1}-Q_{s}^{2}\right|\right) d s .
$$

Then by Gronwall's lemma,

$$
\left|P_{t}^{Q^{1}}-P_{t}^{Q^{2}}\right| \leq 4 \pi\|\beta\|_{1} \exp \left(4 \pi\|\beta\|_{1} t\right) \int_{0}^{t}\left|Q_{s}^{1}-Q_{s}^{2}\right| d s .
$$

Taking $Q_{t}^{1}=Q_{t}^{2}=Q_{t}$ we see that there is a unique probability measure flow solving the linearized equation associated with any $\left(Q_{t}\right)_{t \geq 0}$, which must then be the flow of marginals of $P^{Q}$ generated by (3.2).

We now consider the nonlinear equation (2.3). Uniqueness easily follows from (3.4). Now, the existence of a solution of (2.3) is obtained by a standard Picard argument. Let $P_{t}^{0}=P_{0}$ and for $k \geq 0$, the flow $\left(P_{t}^{k}\right)_{t}$ defined for $\phi \in L^{\infty}\left(\mathbb{R}^{3}\right)$ by

$$
\left\langle\phi, P_{t}^{k+1}\right\rangle=\left\langle\phi, P_{0}\right\rangle+\int_{0}^{t}\left\langle K_{\beta}^{\phi}\left(v, v^{*}\right), P_{s}^{k+1}(d v) P_{s}^{k}\left(d v^{*}\right)\right\rangle d s
$$

Iterations of (3.4) imply that

$$
\left|P_{t}^{k+1}-P_{t}^{k}\right| \leq\left(4 \pi\|\beta\|_{1} e^{4 \pi\|\beta\|_{1}}\right)^{k} \frac{t^{k}}{k !} \sup _{0 \leq s \leq t}\left|P_{s}^{1}-P_{s}^{0}\right| .
$$

Then $\left(P_{t}^{k}\right)_{t}$ converges uniformly on compact sets to $\left(\tilde{P}_{t}\right)_{t}$ solving (2.3).

Let us finally come back to the proof of the existence and uniqueness of a solution of the nonlinear martingale problem. We consider the law $P$ generated by (3.2) with $\left(Q_{t}\right)_{t}$ equal to $\left(\tilde{P}_{t}\right)_{t}$. Then the flow $\left(P_{t}\right)_{t}$ satisfies (3.3) with $\left(Q_{t}\right)_{t}$ equal to $\left(\tilde{P}_{t}\right)_{t}$ as does $\left(\tilde{P}_{t}\right)_{t}$. Then $\left(P_{t}\right)_{t}=\left(\tilde{P}_{t}\right)_{t}$ and $P$ solves the nonlinear martingale problem. Now, if $P^{1}$ and $P^{2}$ are two solutions of the nonlinear martingale problem, they have the same flow of time-marginals $\left(\tilde{P}_{t}\right)_{t}$. Then they are solutions of a (standard) wellposed martingale problem, and are then equal.

By adapting the proof of Desvillettes, [3], Theorem A.1, one can prove

Theorem 3.2. Let us assume that $\int_{0}^{\pi} \beta(\theta) d \theta<+\infty$. Let $f_{0} \geq 0$ be an initial density datum such that

$$
\int_{\mathbb{R}^{3}} f_{0}(v)\left(1+|v|^{2}\right) d v<+\infty .
$$

Then there exists a unique density solution $f^{\beta}(t, v)$ of (2.1) in

$$
L^{\infty}\left(\mathbb{R}+, L^{1}\left(\mathbb{R}^{3},\left(1+|v|^{2}\right) d v\right)\right)
$$

with initial datum $f_{0}$. Moreover, this solution satisfies the conservation of momentum and energy.

Using Remark 2.3 we see that under the assumptions of Theorem 3.2 , the solution $P^{\beta}$ of the martingale problem with initial distribution $P_{0}(d v)=f_{0}(v) d v$ satisfies, for each $t>0, P_{t}^{\beta}(d v)=f^{\beta}(t, v) d v$, where $f^{\beta}$ is defined in Theorem 3.2 . 
3.1. Stochastic approximations in the cutoff case. Still under the cutoff assumption $\int_{0}^{\pi} \beta(\theta) d \theta<+\infty$, we define two different mean-field interacting particle systems which will approximate the nonlinear martingale problem.

Let $v^{n}=\left(v_{1}, \ldots, v_{n}\right)$, the generic point of $\left(\mathbb{R}^{3}\right)^{n}$, and let $\mathbf{e}_{i}: h \in \mathbb{R}^{3} \mapsto \mathbf{e}_{i} . h=$ $(0, \ldots, 0, h, 0, \ldots, 0) \in\left(\mathbb{R}^{3}\right)^{n}$ with $h$ at the $i$-th place. The interacting systems of $n$ particles that we consider are pure-jump Markov processes with values in $\left(\mathbb{R}^{3}\right)^{n}$ and with generators defined for $\phi \in C_{b}\left(\left(\mathbb{R}^{3}\right)^{n}\right)$ by: for the simple mean-field interacting particle system,

$$
\frac{1}{n-1} \sum_{1 \leq i \neq j \leq n} \int_{0}^{2 \pi} \int_{0}^{\pi}\left(\phi\left(v^{n}+\mathbf{e}_{i} \cdot\left(a\left(v_{i}, v_{j}, \theta, \varphi\right)\right)\right)-\phi\left(v^{n}\right)\right) \beta(\theta) d \theta d \varphi ;
$$

for the binary mean-field interacting particle system,

$$
\begin{aligned}
\frac{1}{n-1} \sum_{1 \leq i \neq j \leq n} \int_{0}^{2 \pi} \int_{0}^{\pi} \frac{1}{2}( & \phi\left(v^{n}+\mathbf{e}_{i} \cdot\left(a\left(v_{i}, v_{j}, \theta, \varphi\right)\right)\right. \\
& \left.\left.+\mathbf{e}_{j} \cdot\left(a\left(v_{j}, v_{i}, \theta,-\varphi\right)\right)\right)-\phi\left(v^{n}\right)\right) \beta(\theta) d \theta d \varphi .
\end{aligned}
$$

We will treat these two cases in the same way, since in a probabilistic point of view their behaviours are completely similar. The first particle system can be seen as associated with the Nanbu algorithm (cf. [12, [1]) and is as simple as possible. The second one can be related to the Bird algorithm (cf. [18]). Its real and main interest is that it conserves momentum and kinetic energy. In both cases, we denote by $V^{\beta, n}=\left(V^{\beta, 1 n}, \ldots, V^{\beta, n n}\right)$ this Markov process.

We use a convergence result proved in Graham-Méléard [6], Theorem 3.1 and obtain a strong approximation result. For a given $T>0$, let us denote by $|\cdot|_{T}$ the total variation norm in the space of signed measures on $\mathbb{D}\left([0, T], \mathbb{R}^{3}\right)$. Then we have a propagation of chaos result in a variation norm sense.

Theorem 3.3. Let $\left(V_{0}^{i}\right)_{i \geq 1}$ be independent and $P_{0}$-distributed random variables. For given $T>0$ and $k \in \mathbb{N}^{*}$,

$$
\left|\mathcal{L}\left(V^{\beta, 1 n}, \ldots, V^{\beta, k n}\right)-\left(P^{\beta}\right)^{\otimes k}\right|_{T} \leq K_{k} \frac{\exp \left(2 \pi\|\beta\|_{1} T\right)}{n},
$$

with $K_{1}, K_{2}=6$ and $K_{k}=2 k(k-1)$ for $k>2$.

The following corollary will be the basis for the numerical approximations in the sequel.

Corollary 3.4. The empirical measure defined by

$$
\mu^{\beta, n}=\frac{1}{n} \sum_{i=1}^{n} \delta_{V^{\beta, i n}}
$$

converges in probability in $\mathcal{P}\left(\mathbb{D}\left([0, T], \mathbb{R}^{3}\right)\right)$ to $P^{\beta}$, with the rate $\sqrt{K \frac{\exp \left(2 \pi\|\beta\|_{1} T\right)}{n}}$. (The space $\mathcal{P}\left(\mathbb{D}\left([0, T], \mathbb{R}^{3}\right)\right)$ is endowed with the weak topology for the Skorohod metric on $\left.\mathbb{D}\left([0, T], \mathbb{R}^{3}\right)\right)$.

Then we can prove that under the assumptions of Theorem [3.2] the empirical measure at time $t$ converges for each fixed $t$ to the function $f^{\beta}(t,$.$) solution of the$ 
Boltzmann equation with cutoff. We now consider the case without cutoff which is the original part of the paper.

\section{The Case without CutofF}

In the case without cutoff, the existence and uniqueness of the associated nonlinear martingale problem is not so standard as in the cutoff case. In order to prove the existence, we associate with this martingale problem a nonlinear stochastic differential equation on a greater probability space. We prove the existence of a solution to this stochastic differential equation (SDE) on each finite-time interval $[0, T]$ by a generalized Picard iteration and we deduce the existence of a solution of the nonlinear martingale problem.

Notation 4.1. 1. We consider now two probability spaces: the first one is the abstract space $\left(\Omega, \mathcal{F},\left\{\mathcal{F}_{t}\right\}_{t \in[0, T]}, P\right)$ and the second one is $([0,1], \mathcal{B}([0,1]), d \alpha)$. In order to avoid any confusion, the processes on $([0,1], \mathcal{B}([0,1]), d \alpha)$ will be said to be some $\alpha$-processes, the expectation under $d \alpha$ will be denoted $E_{\alpha}$, and the laws $\mathcal{L}_{\alpha}$.

2. If $Q$ is a probability on $\mathbb{D}_{T}=\mathbb{D}\left([0, T], \mathbb{R}^{3}\right)$, we will say that $Q \in \mathcal{P}_{2}\left(\mathbb{D}_{T}\right)$ if $\int_{x \in \mathbb{D}_{T}} \sup _{[0, T]}|x(t)|^{2} Q(d x)<\infty$. A right-continuous and left-handed limited adapted process $Y_{s}$ on $[0, T]$ will be said to be an $L_{T}^{2}$-process if its law belongs to $\mathcal{P}_{2}\left(\mathbb{D}_{T}\right)$.

Notation 4.2. Let $V_{0}$ be an $\mathbb{R}^{3}$-valued $\mathcal{F}_{0}$-measurable random variable, let $N$ be an $\left\{\mathcal{F}_{t}\right\}$-Poisson measure on $[0, T] \times[0,1] \times[0, \pi] \times[0,2 \pi]$ and $\tilde{N}$ its compensated measure. Let $Y$ be an $L_{T}^{2}$-process, let $W$ be an $L_{T}^{2}$ - $\alpha$-process, and let $b \in \mathbb{R}$. Then we denote by $X=F\left(V_{0}, Y, W, N, b\right)$ the $L_{T}^{2}$-process defined by

$$
\begin{aligned}
X_{t}= & V_{0}+\int_{0}^{t} \int_{0}^{1} \int_{0}^{\pi} \int_{0}^{2 \pi} a\left(Y_{s-}, W_{s-}(\alpha), \theta, \varphi\right) \tilde{N}(d \theta, d \varphi, d \alpha, d s) \\
& -b \int_{0}^{t} \int_{0}^{1}\left(Y_{s-}-W_{s-}(\alpha)\right) d \alpha d s
\end{aligned}
$$

Definition 4.3. We will say that $(V, W, N)$ is a solution of $\operatorname{SDE}\left(\beta, P_{0}\right)$ if:

- $V$ is an $L_{T}^{2}$-process, such that $\mathcal{L}\left(V_{0}\right)=P_{0}$,

- $W$ is an $L_{T}^{2}-\alpha$-process such that $\mathcal{L}_{\alpha}(W)=\mathcal{L}(V)$,

- $N$ is an $\left\{\mathcal{F}_{t}\right\}$-Poisson measure on $[0, T] \times[0,1] \times[0, \pi] \times[0,2 \pi]$ with intensity measure $\beta(\theta) d \theta d \varphi d \alpha d s$ and compensated measure $\tilde{N}$,

- $V=F\left(V_{0}, V, W, N, b\right)$, where $b=\pi \int_{0}^{\pi}(1-\cos \theta) \beta(\theta) d \theta$

Remark 4.4. If $(V, W, N)$ is a solution of $\operatorname{SDE}\left(\beta, P_{0}\right)$, then $\mathcal{L}(V)=\mathcal{L}_{\alpha}(W)$ is a solution of $\operatorname{MP}\left(\beta, P_{0}\right)$, and thus $\left\{\mathcal{L}\left(V_{s}\right)\right\}_{s \in[0, T]}$ is the unique measure-solution of (2.3). (The uniqueness is recalled in Theorem[2.2])

We now state an existence and uniqueness result.

Theorem 4.5. Assume that $P_{0}$ is a probability measure on $\mathbb{R}^{3}$ admitting a moment of order 2 and that $\beta$ is a cross section satisfying $\int_{0}^{\pi} \theta^{2} \beta(\theta) d \theta<\infty$. Then

1. $\operatorname{SDE}\left(\beta, P_{0}\right)$ admits a solution $(V, W, N)$ and the law $P^{\beta}=\mathcal{L}(V)=\mathcal{L}_{\alpha}(W)$ is unique.

2. $\operatorname{MP}\left(\beta, P_{0}\right)$ admits a unique solution given by $P^{\beta}$. 
We first prove the uniqueness for $\operatorname{MP}\left(\beta, P_{0}\right)$, which will also imply the uniqueness in law for $\operatorname{SDE}\left(\beta, P_{0}\right)$. Our proof is an easy corollary of Theorem 2.2 (which is due to Toscani-Villani [17]).

Corollary 4.6. Under the same assumptions as in Theorem 2.2, the uniqueness of a solution to the nonlinear martingale problem $\mathrm{MP}\left(\beta, P_{0}\right)$ holds.

Proof. Let $Q$ be a solution of $\mathrm{MP}\left(\beta, P_{0}\right)$. Then the marginal flow $\left\{Q_{s}\right\}$ is a measure solution of (2.3). We thus know from Theorem 2.2 that for each $s, Q_{s}$ is uniquely determined. Now $Q$ is the law of a Markov process with initial distribution $P_{0}$ and with the given infinitesimal generator

$$
L \phi(v)=\int_{v_{*} \in \mathbb{R}^{3}} K_{\beta}^{\phi}\left(v, v_{*}\right) Q_{s}\left(d v_{*}\right),
$$

and the conclusion follows.

We now want to prove the existence of a solution to $\operatorname{SDE}\left(\beta, P_{0}\right)$. The following lemma is a tricky remark of Tanaka, [16].

Lemma 4.7. Let $N$ be an $\left\{\mathcal{F}_{t}\right\}$-Poisson measure on $[0, T] \times[0,1] \times[0, \pi] \times[0,2 \pi]$ with intensity measure $\beta(\theta) d \theta d \varphi d \alpha d s$ and compensated measure $\tilde{N}$. Let $\varphi_{*}(\omega, s, \alpha)$ be a predictable $\left[0,2 \pi\left[\right.\right.$-valued function. We define a new counting measure $N^{*}$ on $[0, T] \times[0,1] \times[0, \pi] \times[0,2 \pi]$ by

$$
N^{*}(A)=\int 1_{A}\left(s, \alpha, \theta, \varphi+\varphi_{*}(s, \alpha)\right) N(d \theta, d \varphi, d \alpha, d s)
$$

Then $N^{*}$ is again an $\left\{\mathcal{F}_{t}\right\}$-Poisson measure on $[0, T] \times[0,1] \times[0, \pi] \times[0,2 \pi]$ with intensity measure $\beta(\theta) d \theta d \varphi d \alpha d s$. Furthermore, for every predictable function $h(\omega, s, \alpha, \theta, \varphi)$ such that the expressions below are well defined,

$$
\int h(s, \alpha, \theta, \varphi) \tilde{N}^{*}(d \theta, d \varphi, d \alpha, d s)=\int h\left(s, \alpha, \theta, \varphi+\varphi_{*}(s, \alpha)\right) \tilde{N}(d \theta, d \varphi, d \alpha, d s)
$$

The proof of this lemma is immediate. We finally prove the existence of a solution to $\operatorname{SDE}\left(\beta, P_{0}\right)$.

Lemma 4.8. Let $V_{0}$ be an $\mathcal{F}_{0}$-measurable $\mathbb{R}^{3}$-valued random variable belonging to $L^{2}(\Omega)$, and let $\beta$ be a cross section satisfying $\int_{0}^{\pi} \theta^{2} \beta(\theta) d \theta<\infty$. Let $N$ be an $\left\{\mathcal{F}_{t}\right\}$-Poisson measure on $[0, T] \times[0,1] \times[0, \pi] \times[0,2 \pi]$ with intensity measure $\beta(\theta) d \theta d \varphi d \alpha d s$. Then there exists an $L_{T}^{2}$-process $V$, an $L_{T}^{2}$ - $\alpha$-process $W$, and a predictable function $\varphi_{*}(\omega, s, \alpha)$ with values in $\left[0,2 \pi\left[\right.\right.$, such that, if $N^{*}$ is the Poisson measure on $[0, T] \times[0,1] \times[0, \pi] \times[0,2 \pi]$ defined by [4.3), then

$$
V=F\left(V_{0}, V, W, N^{*}, b\right) \quad \text { and } \quad \mathcal{L}(V)=\mathcal{L}_{\alpha}(W)
$$

where $b=\pi \int_{0}^{\pi}(1-\cos \theta) d \theta$. This of course implies the existence of a solution $\left(V, W, N^{*}\right)$ to $\operatorname{SDE}\left(\beta, \mathcal{L}\left(V_{0}\right)\right)$, and thus the existence of a solution to $\operatorname{MP}\left(\beta, \mathcal{L}\left(V_{0}\right)\right)$.

Proof. Following again [16], we use a Picard iteration. First, we set $V^{0} \equiv V_{0}$, and we consider an $\alpha$-process $W^{0}$ such that $\mathcal{L}_{\alpha}\left(W^{0}\right)=\mathcal{L}\left(V^{0}\right)$. We also set $\varphi_{*}^{0} \equiv 0$. 
Then, when everything is built up to $n$, we set

$$
\begin{aligned}
& V^{n+1}= V_{0}+\int_{0}^{t} \int_{0}^{1} \int_{0}^{\pi} \int_{0}^{2 \pi} a\left(V_{s-}^{n}, W_{s-}^{n}(\alpha), \theta, \varphi+\varphi_{*}^{n}(s, \alpha)\right) \tilde{N}(d \theta, d \varphi, d \alpha, d s) \\
&(4.6) \quad-b \int_{0}^{t} \int_{0}^{1}\left(V_{s-}^{n}-W_{s-}^{n}(\alpha)\right) d \alpha d s
\end{aligned}
$$

and we consider an $\alpha$-process $W^{n+1}$ such that $\mathcal{L}_{\alpha}\left(W^{n+1}, \ldots, W^{0}\right)=\mathcal{L}\left(V^{n+1}, \ldots, V^{0}\right)$. We also set $\varphi_{*}^{n+1}(s, \alpha)=\varphi_{*}^{n}(s, \alpha)+\varphi_{0}\left(V_{s-}^{n}-W_{s-}^{n}(\alpha), V_{s-}^{n+1}-W_{s-}^{n+1}(\alpha)\right)$, where the map $\varphi_{0}$ was defined in Lemma 2.6 Then one easily checks, thanks to Doob's inequality, Lemma 2.6. and since $\mathcal{L}_{\alpha}\left(W^{n}, W^{n-1}\right)=\mathcal{L}\left(V^{n}, V^{n-1}\right)$, that

$$
\begin{aligned}
E\left(\sup _{[0, t]}\left|V_{s}^{n+1}-V_{s}^{n}\right|^{2}\right) & \leq K \int_{0}^{t}\left[E\left(\left|V_{s}^{n}-V_{s}^{n-1}\right|^{2}\right)+E_{\alpha}\left(\left|W_{s}^{n}-W_{s}^{n-1}\right|^{2}\right)\right] d s \\
& \leq K \int_{0}^{t} E\left(\left|V_{s}^{n}-V_{s}^{n-1}\right|^{2}\right) d s
\end{aligned}
$$

Thus there clearly exist some $L_{T}^{2}$-process $V$, some $L_{T}^{2}$ - $\alpha$-process $W$, such that $\mathcal{L}(V)=\mathcal{L}_{\alpha}(W)$ and when $n$ goes to infinity,

$$
E\left(\sup _{[0, t]}\left|V_{s}-V_{s}^{n}\right|^{2}\right)=E_{\alpha}\left(\sup _{[0, t]}\left|W_{s}-W_{s}^{n}\right|^{2}\right) \longrightarrow 0 .
$$

We still have to study the convergence of $a\left(V_{s-}^{n}, W_{s-}^{n}(\alpha), \theta, \varphi+\varphi_{*}^{n}(s, \alpha)\right)$. First recall that

$$
\begin{aligned}
& a\left(V_{s-}^{n}, W_{s-}^{n}(\alpha), \theta, \varphi+\varphi_{*}^{n}(s, \alpha)\right) \\
& \quad=\frac{\cos \theta-1}{2}\left(V_{s-}^{n}-W_{s-}^{n}(\alpha)\right)+\frac{\sin \theta}{2} \Gamma\left(V_{s-}^{n}-W_{s-}^{n}(\alpha), \varphi+\varphi_{*}^{n}(s, \alpha)\right) .
\end{aligned}
$$

Furthermore, we know from Lemma 2.6 that

$$
\left|\Gamma\left(V_{s-}^{n+1}-W_{s-}^{n+1}(\alpha), \varphi+\varphi_{*}^{n+1}(s, \alpha)\right)-\Gamma\left(V_{s-}^{n}-W_{s-}^{n}(\alpha), \varphi+\varphi_{*}^{n}(s, \alpha)\right)\right|
$$

$$
\leq 3\left(\left|V_{s-}^{n+1}-V_{s-}^{n}(\alpha)\right|+\left|W_{s-}^{n+1}(\alpha)-W_{s-}^{n}(\alpha)\right|\right) .
$$

We thus deduce from (4.8) the existence of a predictable function $\delta(s, \varphi, \alpha)$, such that a.s., for almost all $\alpha$, when $n$ tends to infinity,

$$
\Gamma\left(V_{s-}^{n}-W_{s-}^{n}(\alpha), \varphi+\varphi_{*}^{n}(s, \alpha)\right) \longrightarrow \delta(s, \varphi, \alpha) .
$$

It is clear that $\delta(s, 0, \alpha)$ has the norm $\left|V_{s-}-W_{s-}(\alpha)\right|$ and is orthogonal to $V_{s-}-$ $W_{s-}(\alpha)$. Thus, for some $\varphi_{*}(s, \alpha)$ (which is predictable),

$$
\delta(s, 0, \alpha)=\Gamma\left(V_{s-}-W_{s-}(\alpha), \varphi_{*}(s, \alpha)\right) .
$$

It is not difficult to deduce that for all $\varphi$,

$$
\delta(s, \varphi, \alpha)=\Gamma\left(V_{s-}-W_{s-}(\alpha), \varphi+\varphi_{*}(s, \alpha)\right) .
$$

We thus have found a predictable function $\varphi_{*}$ such that if $N^{*}$ is defined by (4.3), then $V=F\left(V_{0}, V, W, N^{*}, b\right)$. Lemma 4.8 is proved. The proof of Theorem 4.5 is complete. 


\section{Stochastic Particle approximations FOR THE BOLTZMANN EQUATION WITHOUT CUTOFF}

We still consider in this section the Maxwell Boltzmann equation when only $\int_{0}^{\pi} \theta^{2} \beta(\theta) d \theta$ is finite.

We want to approximate the solution of the nonlinear martingale problem $\operatorname{MP}\left(\beta, P_{0}\right)$ on $\mathbb{D}\left([0, T], \mathbb{R}^{3}\right)$ by using a simulable interacting particle system. As an intermediate step, we introduce cutoff approximations of the case without cutoff.

5.1. Convergence of some cutoff approximations. Let us consider the cutoff cross sections $\beta_{l}$ defined by

$$
\beta_{l}(\theta)=\beta(\theta) 1_{\left[\frac{1}{l}, \pi\right]}(\theta)
$$

We are interested in the convergence of the solution $P^{\beta_{l}}$ of the martingale problem on $\mathbb{D}\left([0, T], \mathbb{R}^{3}\right)$ with cross section $\beta_{l}$ to that of the martingale problem $P^{\beta}$ with cross section $\beta$ on $\mathbb{D}\left([0, T], \mathbb{R}^{3}\right)$. Usually (for example for a similar problem in dimension one [4]), one constructs a pathwise coupling of processes, respectively with laws $P^{\beta_{l}}$ and $P^{\beta}$, which we are able to compare in a pathwise sense. Unfortunately, in our situation, the bad behaviour of the function $a$ prevents using this method. So we will construct a sort of "coupling in law".

Notation 5.1. We consider on the set $\mathcal{P}_{2}\left(\mathbb{R}^{3}\right)$ the Vaserstein metric :

$$
\begin{aligned}
& \rho\left(q_{1}, q_{2}\right)=\inf \left\{\int_{\mathbb{R}^{3} \times \mathbb{R}^{3}}|x-y|^{2} r(d x, d y) / \begin{array}{l}
r \in \mathcal{P}_{2}\left(\mathbb{R}^{3} \times \mathbb{R}^{3}\right), \text { and } \\
r \text { has marginals } q_{1} \text { and } q_{2}
\end{array}\right\}^{1 / 2} \\
& (5.2) \quad=\inf \left\{E\left(|V-W|^{2}\right)^{1 / 2} / \mathcal{L}(V)=q_{1}, \mathcal{L}(W)=q_{2}\right\} .
\end{aligned}
$$

Similarly, we also consider the Vaserstein metric on $\mathcal{P}_{2}\left(\mathbb{D}_{T}\right)$.

$$
\begin{aligned}
& \rho_{T}\left(Q_{1}, Q_{2}\right) \\
& \quad=\inf \left\{\int_{\mathbb{D}_{T} \times \mathbb{D}_{T}}\|x-y\|_{\infty}^{2} R(d x, d y) / \begin{array}{l}
R \in \mathcal{P}_{2}\left(\mathbb{D}_{T} \times \mathbb{D}_{T}\right), \text { and } \\
R \text { has marginals } Q_{1} \text { and } Q_{2}
\end{array}\right\}^{1 / 2} \\
& \quad=\inf \left\{E\left(\sup _{[0, T]}\left|V_{t}-W_{t}\right|^{2} / \mathcal{L}(V)=Q_{1}, \mathcal{L}(W)=Q_{2}\right\} .\right.
\end{aligned}
$$

We set

$$
\begin{array}{cc}
c=\int_{0}^{\pi} \theta^{2} \beta(\theta) d \theta, \quad c_{l}=\int_{1 / l}^{\pi} \theta^{2} \beta(\theta) d \theta, \\
b=\pi \int_{0}^{\pi}(1-\cos \theta) \beta(\theta) d \theta, \quad b_{l}=\pi \int_{1 / l}^{\pi}(1-\cos \theta) \beta(\theta) d \theta .
\end{array}
$$

We also define

$$
A_{l}=\left(32 \pi\left(c-c_{l}\right)+4 T\left(b-b_{l}\right)^{2}\right) T .
$$

Let us remark that $A_{l}$ tends to 0 when $l$ tends to infinity. 
Theorem 5.2. Let $P_{0} \in \mathcal{P}_{2}\left(\mathbb{R}^{3}\right)$. Let $\beta$ be a cross section satisfying $\int_{0}^{\pi} \theta^{2} \beta(\theta) d \theta<$ $\infty$, and let $\beta_{l}(\theta)=\beta(\theta) 1_{[1 / l, \pi]}(\theta)$. Let $P^{\beta}$ (resp. $P^{\beta_{l}}$ ) be the unique solution of $\operatorname{MP}\left(\beta, P_{0}\right)$ (resp. $\left.\operatorname{MP}\left(\beta_{l}, P_{0}\right)\right)$. Then

$$
\rho_{T}\left(P^{\beta}, P^{\beta_{l}}\right) \leq K(b, c, T)\left(\int_{\mathbb{R}^{3}}|v|^{2} P_{0}(d v)\right)^{1 / 2} \sqrt{A_{l}}
$$

where the explicit expression of $K(b, c, T)$ is given by

$$
K(b, c, T)=\sqrt{K^{\left[T / \tau_{0}\right]+1}-1}
$$

and

$$
K=10 e^{5\left(16 \pi c+2 b^{2} T\right)} ; \quad \tau_{0}=\frac{1}{K\left(16 \pi c+2 b^{2} T\right)}=\frac{1}{10} \frac{e^{-5\left(16 \pi c+2 b^{2} T\right) T}}{16 \pi c+2 b^{2} T},
$$

and $\left[T / \tau_{0}\right]$ denotes the integer part of $T / \tau_{0}$.

In order to prove this result, we begin with a lemma whose proof is very similar (but much more standard) to those of Lemma 4.8 and Corollary 4.6 and thus is omitted.

Lemma 5.3. Let $V_{0}$ be a $\mathbb{R}^{3}$-valued $\mathcal{F}_{0}$-measurable random variable such that $E\left(\left|V_{0}\right|^{2}\right)<\infty$, and let $\beta$ be a cross section satisfying $\int_{0}^{\pi} \theta^{2} \beta(\theta) d \theta<\infty$. Let $N$ be an $\left\{\mathcal{F}_{t}\right\}$-Poisson measure on $[0, T] \times[0,1] \times[0, \pi] \times[0,2 \pi]$ with intensity measure $\beta(\theta) d \theta d \varphi d \alpha d s$, and let $Y$ be an $L_{T}^{2}-\alpha$-process.

Then there exist an $L_{T}^{2}$-process $V$ and a predictable function $\varphi_{*}(\omega, s, \alpha)$ with values in $\left[0,2 \pi\left[\right.\right.$, such that, if $N^{*}$ is the Poisson measure on $[0, T] \times[0,1] \times[0, \pi] \times$ $[0,2 \pi]$ defined by (4.3), then

$$
V=F\left(V_{0}, V, Y, N^{*}, b\right) .
$$

The law $\mathcal{L}(V)$ is unique and entirely determined by $\mathcal{L}\left(V_{0}\right), \beta$, and the marginal flow $\left\{\mathcal{L}_{\alpha}\left(Y_{s}\right)\right\}_{s \in[0, T]}$.

The proof of Theorem 5.2 is based on the following lemma, which begins a recursive proof.

Lemma 5.4. Let $P_{0}$ and $P_{0}^{\prime}$ belong to $\mathcal{P}_{2}\left(\mathbb{R}^{3}\right)$. Let $\beta$ be a cross section satisfying $\int_{0}^{\pi} \theta^{2} \beta(\theta) d \theta<\infty$, and let $\beta_{l}(\theta)=\beta(\theta) 1_{[1 / l, \pi]}(\theta)$. Let $P^{\beta}$ and $P^{\beta_{l}}$ be the unique solutions of $\operatorname{MP}\left(\beta, P_{0}\right)$ and $\operatorname{MP}\left(\beta_{l}, P_{0}^{\prime}\right)$. Then, for $\tau_{0}$ and $K$ defined by (5.6),

$$
\rho_{\tau_{0}}^{2}\left(P^{\beta}, P^{\beta_{l}}\right) \leq K\left(\rho^{2}\left(P_{0}, P_{0}^{\prime}\right)+\left\{\int_{\mathbb{R}^{3}}|v|^{2} P_{0}(d v)\right\} A_{l}\right) .
$$

Proof. Let $\epsilon>0, \tau>0$, and $l \geq 1$ be fixed. We consider two random variables $V_{0}$ and $V_{0}^{\prime}$ with laws given by $P_{0}$ and $P_{0}^{\prime}$ and such that

$$
E\left(\left|V_{0}-V_{0}^{\prime}\right|^{2}\right) \leq \rho^{2}\left(P_{0}, P_{0}^{\prime}\right)+\epsilon .
$$

We also consider some $L_{T}^{2}-\alpha$-processes $W^{\epsilon}$ and $W^{l, \epsilon}$ with laws $P^{\beta}$ and $P^{\beta_{l}}$ and such that

$$
E_{\alpha}\left(\sup _{[0, \tau]}\left|W_{s}^{\epsilon}-W_{s}^{l, \epsilon}\right|^{2}\right) \leq \rho_{\tau}^{2}\left(P^{\beta}, P^{\beta_{l}}\right)+\epsilon .
$$

Let $N$ be an $\left\{\mathcal{F}_{t}\right\}$-Poisson measure on $[0, T] \times[0,1] \times[0, \pi] \times[0,2 \pi]$ with intensity measure $\beta(\theta) d \theta d \varphi d \alpha d s$. Thanks to Lemma [5.3, there exist a $L_{T}^{2}$-process $Z^{\epsilon}$ and a 
predictable function $\varphi_{*}^{\epsilon}(\omega, s, \alpha)$ with values in $\left[0,2 \pi\left[\right.\right.$ such that, if $N_{\epsilon}^{*}$ is the $\left\{\mathcal{F}_{t}\right\}$ Poisson measure on $[0, T] \times[0,1] \times[0, \pi] \times[0,2 \pi]$ defined by (4.3) (with $\varphi_{*}^{\epsilon}$ instead of $\left.\varphi_{*}\right)$, then

$$
Z^{\epsilon}=F\left(V_{0}, Z^{\epsilon}, W^{\epsilon}, N_{\epsilon}^{*}, b\right)
$$

Let us prove that $\mathcal{L}\left(Z^{\epsilon}\right)=\mathcal{L}_{\alpha}\left(W^{\epsilon}\right)=P^{\beta}$. Thanks to Lemma 5.3 we know that the law of $Z^{\epsilon}$ is uniquely determined by $\beta, P_{0}$, and $\mathcal{L}_{\alpha}\left(W^{\epsilon}\right)$. On the other hand, if $(V, W, \hat{N})$ is any solution of $\operatorname{SDE}\left(\beta, P_{0}\right)$ with $W$ fixed with $\mathcal{L}_{\alpha}(W)=\mathcal{L}_{\alpha}\left(W^{\epsilon}\right)=P^{\beta}$, $V$ can be considered as a solution of the classical stochastic differential equation studied in Lemma 5.3. The uniqueness in law in (5.11) implies that the law of $Z^{\epsilon}$ is the same as that of $V$, and thus that $\mathcal{L}\left(Z^{\epsilon}\right)=\mathcal{L}_{\alpha}\left(W^{\epsilon}\right)=P^{\beta}$.

We now set $N_{\epsilon, l}^{*}=\left.N_{\epsilon}^{*}\right|_{[0, T] \times[0,1] \times[1 / l, \pi] \times[0,2 \pi]}$. Since $\int_{1 / l}^{\pi} \beta(\theta) d \theta<\infty, N_{\epsilon, l}^{*}$ is a finite Poisson measure, thus it can a.s. be written as

$$
N_{\epsilon, l}^{*}=\sum_{i=1}^{\mu} \delta_{\left(T_{i}, \alpha_{i}, \theta_{i}, \varphi_{i}\right)} .
$$

Working recursively on the time intervals $\left[T_{i}, T_{i+1}\left[\right.\right.$, one can easily build an $L_{T^{-}}^{2}$ process $Z^{\epsilon, l}$ such that

$$
\begin{gathered}
Z_{t}^{\epsilon, l}=V_{0}^{\prime}+\int_{0}^{t} \int_{0}^{1} \int_{0}^{\pi} \int_{0}^{2 \pi} a\left(Z_{s-}^{\epsilon, l}, W_{s-}^{\epsilon, l}(\alpha), \theta, \varphi\right. \\
\left.+\varphi_{0}\left(Z_{s-}^{\epsilon}-W_{s-}^{\epsilon}(\alpha), Z_{s-}^{\epsilon, l}-W_{s-}^{\epsilon, l}(\alpha)\right)\right) \\
N_{\epsilon, l}^{*}(d \theta, d \varphi, d \alpha, d s)
\end{gathered}
$$

Indeed, it suffices to set $Z_{t}^{\epsilon, l}=V_{0}^{\prime}$ for all $t \in\left[0, T_{1}[\right.$, then to set

$$
\begin{aligned}
Z_{t}^{\epsilon, l}=V_{0}^{\prime}+a\left(Z_{T_{1}-}^{\epsilon, l}, W_{T_{1}-}^{\epsilon, l}\right. & \left(\alpha_{1}\right), \theta_{1}, \varphi_{1} \\
& \left.+\varphi_{0}\left(Z_{T_{1}-}^{\epsilon}-W_{T_{1}-}^{\epsilon}\left(\alpha_{1}\right), Z_{T_{1}-}^{\epsilon, l}-W_{T_{1}-}^{\epsilon, l}\left(\alpha_{1}\right)\right)\right)
\end{aligned}
$$

for all $t \in\left[T_{1}, T_{2}\right.$ [, etc. (No Picard iteration is needed here.) Notice that (5.13) can be rewritten with respect to the compensated measure as

$$
\begin{gathered}
Z_{t}^{\epsilon, l}=V_{0}^{\prime}-b_{l} \int_{0}^{t} \int_{0}^{1}\left(Z_{s-}^{\epsilon, l}-W_{s-}^{\epsilon, l}\right) d \alpha d s \\
+\int_{0}^{t} \int_{0}^{1} \int_{1 / l}^{\pi} \int_{0}^{2 \pi} a\left(Z_{s-}^{\epsilon, l}, W_{s-}^{\epsilon, l}(\alpha), \theta, \varphi\right. \\
\left.+\varphi_{0}\left(Z_{s-}^{\epsilon}-W_{s-}^{\epsilon}(\alpha), Z_{s-}^{\epsilon, l}-W_{s-}^{\epsilon, l}(\alpha)\right)\right) \\
\tilde{N}_{\epsilon}^{*}(d \theta, d \varphi, d \alpha, d s) .
\end{gathered}
$$

As previously and thanks to Lemma [5.3] we see that $\mathcal{L}\left(Z^{\epsilon, l}\right)=\mathcal{L}_{\alpha}\left(W^{\epsilon, l}\right)=P^{\beta_{l}}$. 
A standard computation, using Doob's inequality and Lemma 2.6, shows that

$$
\begin{aligned}
& E\left(\sup _{[0, t]}\left|Z_{s}^{\epsilon}-Z_{s}^{\epsilon, l}\right|^{2}\right) \leq 5 E\left(\left|V_{0}-V_{0}^{\prime}\right|^{2}\right) \\
& \quad+5\left(16 \pi c+2 b^{2} T\right) \int_{0}^{t}\left(E\left(\left|Z_{s}^{\epsilon}-Z_{s}^{\epsilon, l}\right|^{2}\right)+E_{\alpha}\left(\left|W_{s}^{\epsilon}-W_{s}^{\epsilon, l}\right|^{2}\right)\right) d s \\
& \quad+5\left\{16 \pi\left(c-c_{l}\right)+2 T\left(b-b_{l}\right)^{2}\right\} \int_{0}^{t}\left(E\left(\left|Z_{s}^{\epsilon}\right|^{2}\right)+E_{\alpha}\left(\left|W_{s}^{\epsilon}\right|^{2}\right)\right) d s .
\end{aligned}
$$

Since $\mathcal{L}\left(Z^{\epsilon}\right)=\mathcal{L}_{\alpha}\left(W^{\epsilon}\right)=P^{\beta}$ and since $\left\{P_{s}^{\beta}\right\}_{[0, T]}$ is a measure solution of (2.3), it is well known that for all $s$, we have the conservation of the kinetic energy:

$$
E\left(\left|Z_{s}^{\epsilon}\right|^{2}\right)=E_{\alpha}\left(\left|W_{s}^{\epsilon}\right|^{2}\right)=\int_{\mathbb{R}^{3}}|v|^{2} P_{s}^{\beta}(d v)=\int_{\mathbb{R}^{3}}|v|^{2} P_{0}(d v)=E\left(\left|V_{0}\right|^{2}\right) .
$$

Hence, using Gronwall's Lemma, (15.9) and (15.10), we deduce from (15.16) that

$$
\begin{aligned}
E\left(\sup _{[0, \tau]}\left|Z_{s}^{\epsilon}-Z_{s}^{\epsilon, l}\right|^{2}\right) & \\
\leq 5 e^{5\left(16 \pi c+2 b^{2} T\right) T}\left(\rho^{2}\left(P_{0}, P_{0}^{\prime}\right)\right. & +\epsilon+\left(16 \pi c+2 b^{2} T\right) \tau\left(\rho_{\tau}^{2}\left(P^{\beta}, P^{\beta_{l}}\right)+\epsilon\right) \\
& \left.+\left(32 \pi\left(c-c_{l}\right)+4 T\left(b-b_{l}\right)^{2}\right) T E\left(\left|V_{0}\right|^{2}\right)\right) \\
\leq & \frac{K}{2}\left(\rho^{2}\left(P_{0}, P_{0}^{\prime}\right)+\epsilon+\left(16 \pi c+2 b^{2} T\right) \tau\left(\rho_{\tau}^{2}\left(P^{\beta}, P^{\beta_{l}}\right)+\epsilon\right)+A_{l} E\left(\left|V_{0}\right|^{2}\right)\right) .
\end{aligned}
$$

Using the fact that for all $\epsilon>0$,

$$
\rho_{\tau}^{2}\left(P^{\beta}, P^{\beta_{l}}\right) \leq E\left(\sup _{[0, \tau]}\left|Z_{t}^{\epsilon}-Z_{t}^{\epsilon, l}\right|^{2}\right)
$$

we deduce, making $\epsilon$ go to 0 , and choosing $\tau_{0}=\frac{1}{K\left(16 \pi c+2 b^{2} T\right)}=\frac{1}{10} \frac{e^{-5\left(16 \pi c+2 b^{2} T\right) T}}{16 \pi c+2 b^{2} T}$, that

$$
\rho_{\tau_{0}}^{2}\left(P^{\beta}, P^{\beta_{l}}\right) \leq K\left(\rho^{2}\left(P_{0}, P_{0}^{\prime}\right)+A_{l} E\left(\left|V_{0}\right|^{2}\right)\right)
$$

which was our aim.

Proof of Theorem 5.2. We work recursively on the time intervals $\left[n \tau_{0},(n+1) \tau_{0}\right]$, for $n \leq T / \tau_{0}-1$. First of all, it is clear, from Lemma 5.4, that

$$
\rho_{\tau_{0}}^{2}\left(P^{\beta}, P^{\beta_{l}}\right) \leq K\left\{\int_{\mathbb{R}^{3}}|v|^{2} P_{0}(d v)\right\} A_{l}
$$

where $K=10 e^{5\left(16 \pi c+2 b^{2} T\right) T}$. Now let $1 \leq n \leq T / \tau_{0}-1$ be fixed. Then in particular

$$
\rho^{2}\left(P_{n \tau_{0}}^{\beta}, P_{n \tau_{0}}^{\beta_{l}}\right) \leq \rho_{n \tau_{0}}^{2}\left(P^{\beta}, P^{\beta_{l}}\right) .
$$

On the other hand, an immediate adaptation of Lemma 5.4 shows that

$$
\rho_{n \tau_{0},(n+1) \tau_{0}}^{2}\left(P^{\beta}, P^{\beta_{l}}\right) \leq K\left\{\rho^{2}\left(P_{n \tau_{0}}^{\beta}, P_{n \tau_{0}}^{\beta_{l}}\right)+\int_{\mathbb{R}^{3}}|v|^{2} P_{0}(d v) \times A_{l}\right\}
$$


where

$$
\begin{aligned}
& \rho_{n \tau_{0},(n+1) \tau_{0}}\left(P^{\beta}, P^{\beta_{l}}\right) \\
& =\inf \left\{E\left(\sup _{t \in\left[n \tau_{0},(n+1) \tau_{0}\right]}\left|X_{t}-Y_{t}\right|^{2}\right)^{1 / 2} / \begin{array}{c}
\mathcal{L}(X)=P^{\beta} \\
\mathcal{L}(Y)=P^{\beta_{l}}
\end{array}\right\} .
\end{aligned}
$$

Finally, using the fact that

$$
\rho_{(n+1) \tau_{0}}^{2}\left(P^{\beta}, P^{\beta_{l}}\right) \leq \rho_{n \tau_{0}}^{2}\left(P^{\beta}, P^{\beta_{l}}\right)+\rho_{n \tau_{0},(n+1) \tau_{0}}^{2}\left(P^{\beta}, P^{\beta_{l}}\right)
$$

we obtain

$$
\rho_{(n+1) \tau_{0}}^{2}\left(P^{\beta}, P^{\beta_{l}}\right) \leq(K+1) \rho_{n \tau_{0}}^{2}\left(P^{\beta}, P^{\beta_{l}}\right)+K \int_{\mathbb{R}^{3}}|v|^{2} P_{0}(d v) \times A_{l} .
$$

By standard arguments, we obtain immediately that

$$
\begin{aligned}
\rho_{T}^{2}\left(P^{\beta}, P^{\beta_{l}}\right) & \leq \frac{(K+1)^{\left[T / \tau_{0}\right]}-1}{(K+1)-1} K\left\{\int_{\mathbb{R}^{3}}|v|^{2} P_{0}(d v)\right\} A_{l} \\
& =\left((K+1)^{\left[T / \tau_{0}\right]}-1\right)\left\{\int_{\mathbb{R}^{3}}|v|^{2} P_{0}(d v)\right\} A_{l}
\end{aligned}
$$

where $\left[T / \tau_{0}\right]$ denotes the integer part of $T / \tau_{0}$.

5.2. Convergence rates for the interacting particle systems. We consider the same cutoff cross section $\beta_{l}$ as before. Then with each $l$, one can associate a particle system $\left(V^{\beta_{l}, n}\right)$ as defined in subsection 2.3 .

We can now state our main pathwise convergence result.

Theorem 5.5. Let $\beta$ be a cross section. Let us consider a sequence $l(n)$ of integers going to infinity in such a way that

$$
\exp \left(2 \pi T\left\|\beta_{l(n)}\right\|_{1}\right)=o(n)
$$

and let $\left(V_{0}^{i}\right)_{i \in \mathbb{N}}$ be i.i.d. $P_{0}$-distributed random variables. Then

1. For every fixed $k$ and every $T>0$, the sequence of laws

$$
\mathcal{L}\left(V^{\beta_{l(n)}, 1 n}, \ldots, V^{\beta_{l(n)}, k n}\right)
$$

of probability measures on the path space $\mathbb{D}\left([0, T],\left(\mathbb{R}^{3}\right)^{k}\right)$ converges weakly to $\left(P^{\beta}\right)^{\otimes k}$, where $P^{\beta}$ is the unique solution of $\operatorname{MP}\left(\beta, P_{0}\right)$. Moreover, we have the convergence estimates

$$
\begin{aligned}
& \sup _{0 \leq t \leq T} \rho^{2}\left(\mathcal{L}\left(V_{t}^{\beta_{l(n)}, k n}\right), P_{t}^{\beta}\right) \leq \rho_{T}^{2}\left(\mathcal{L}\left(V^{\beta_{l(n)}, k n}\right), P^{\beta}\right) \\
& \leq 6 \frac{\exp \left(2 \pi T\left\|\beta_{l(n)}\right\|_{1}\right)}{n}+K(b, c, T) A_{l(n)} \int|v|^{2} P_{0}(d v)
\end{aligned}
$$

where the explicit forms of $K(b, c, T)$ and $A_{l}$ are given in subsection 5.1. Recall that $A_{l(n)}$ tends to 0 as $n$ tends to infinity.

2. The empirical measures of the system $\mu^{\beta_{l(n)}, n}$ (defined by (3.8)) converge in probability to $P^{\beta}$ in the path space $\mathcal{P}\left(\mathbb{D}\left([0, T], \mathbb{R}^{3}\right)\right)$. 
The proof is immediate by associating Theorem 3.3 and Theorem 5.2 ,

Let us notice that in the case of potential interactions in $1 / r^{s}, s>2$, i.e. when the cross section is smaller than $C \theta^{-\alpha}$ with $\left.\alpha=\frac{s+1}{s-1} \in\right] 1,3[$, the sequence $l(n)$ has to be chosen in such a way that

$$
\exp \left(2 \pi T \frac{C}{\alpha-1} l(n)^{\alpha-1}\right)=o(n)
$$

\section{The SimUlation ALgORITHMS}

We deduce from the above study two algorithms associated respectively with the simple mean-field ("Nanbu" approach) interacting particle system and the binary mean-field ("Bird" approach) interacting particle system. The description of the algorithms is the same in both cases since the theoretical justification is unified for the two systems.

From now on, the cross section $\beta$, the initial distribution $P_{0}$, the terminal time $T>0$, the size of the particle system $n \geq 2$, and the cutoff parameter $l>0$ are fixed. We denote by $\beta_{l}$ the corresponding cross section with cutoff defined by (5.1). We consider the associated Nanbu and Bird particle systems, $\left\{V_{t}^{N}\right\}_{t \in[0, T]} \in \mathbb{D}\left([0, T],\left(\mathbb{R}^{3}\right)^{n}\right)$ and $\left\{V_{t}^{B}\right\}_{t \in[0, T]} \in \mathbb{D}\left([0, T],\left(\mathbb{R}^{3}\right)^{n}\right)$, which were both denoted $V^{\beta_{l}, n}$ previously. Recall that for each $i \in\{1, \ldots, n\}, V^{N, i}$ (or $V^{B, i}$ ) describes the evolution of the velocity of the $i$-th particle. Our aim is to describe algorithms to simulate $V^{N}$ and $V^{B}$. Of course these algorithms can be derived immediately from (3.6) and (3.7).

First, we assume that $V_{0}^{N}\left(\right.$ or $\left.V_{0}^{B}\right)$ is simulated, according a $P_{0}^{\otimes n}$-distribution. Then we denote by $0<T_{1}^{N}<\ldots<T_{\mu_{N}}^{N}$ (resp. $0<T_{1}^{B}<\ldots<T_{\mu_{B}}^{B}$ ) the successive times of jump until $T$ of a standard Poisson process with parameter $2 \pi n\left\|\beta_{l}\right\|_{1}$ (resp. $2 \pi n\left\|\beta_{l}\right\|_{1} / 2$ ). These times model the times of collision (either there is a direct procedure to simulate the collision times of this process during an a priori chosen time interval, or one simulates independent exponential laws with this rate which describe the inter-collision time intervals).

Before the first collision, the velocities do not change, so that we set $V_{s}^{N}=V_{0}^{N}$ for all $s<T_{1}^{N}$ (resp. $V_{s}^{B}=V_{0}^{B}$ for all $s<T_{1}^{B}$ ). Let us describe the first collision. First, we choose a couple $(i, j)$ of particles, according to a uniform distribution on $\left\{(k, l) \in\{1, \ldots, n\}^{2} \mid k \neq l\right\}$. We choose the first angle $\varphi$ of collision by using a uniform distribution over $[0,2 \pi]$. We finally choose the second angle of collision $\theta$ by using a $\frac{\beta_{l}(\theta)}{\|\beta\|_{1}} d \theta$-distribution. Then we set

$$
\begin{aligned}
V_{T_{1}}^{N, i} & =V_{0}^{N, i}+a\left(V_{0}^{N, i}, V_{0}^{N, j}, \theta, \varphi\right) \\
V_{T_{1}}^{N, k} & =V_{0}^{N, k} \text { if } k \neq i
\end{aligned}
$$

for the simulation of the Nanbu system, and

$$
\begin{aligned}
V_{T_{1}}^{B, i} & =V_{0}^{B, i}+a\left(V_{0}^{B, i}, V_{0}^{B, j}, \theta, \varphi\right) \\
V_{T_{1}}^{B, j} & =V_{0}^{B, j}+a\left(V_{0}^{B, j}, V_{0}^{B, i}, \theta,-\varphi\right) \\
V_{T_{1}}^{B, k} & =V_{0}^{B, k} \text { if } k \notin\{i, j\}
\end{aligned}
$$

in the case of the Bird system. 
Since nothing happens between $T_{1}$ and $T_{2}$, we set $V_{s}^{N}=V_{T_{1}}^{N}$ for all $s \in\left[T_{1}^{N}, T_{2}^{N}\right.$ [ (resp. $V_{s}^{B}=V_{T_{1}}^{B}$ for all $s \in\left[T_{1}^{B}, T_{2}^{B}[\right.$ ).

Iterating this method, we can simulate $V_{T_{2}^{N}}^{N}, \ldots, V_{T_{\mu_{N}}^{N}}^{N}$ (resp. $\left.V_{T_{2}^{B}}^{B}, \ldots, V_{T_{\mu_{B}}^{B}}^{B}\right)$, i.e. the whole path $\left\{V_{t}^{N}\right\}_{t \in[0, T]}$ (resp. $\left\{V_{t}^{B}\right\}_{t \in[0, T]}$ ), which was our aim.

These algorithms are very simple, take a few lines of program and do not require to discretize time.

Notice that in the case of the Nanbu approach, we are far from simulating a gas: in the collisions between two particles, we do only change the velocity of one particle. However, the most "probabilistically" natural system is obtained by this approach, since the martingale problem (2.4) leads immediately to (3.6), and thus to this algorithm.

Of course, Bird's system is more physically natural, since in this case, we simulate binary interactions, and since it preserves momentum and kinetic energy.

\section{NumericAl RESUlts}

We are now interested in numerical results obtained by the simulations algorithms described previously. In order to test these algorithms, we choose a typical cross section without cutoff, which does not admit a moment of order 1:

$$
\beta(\theta)=\frac{1}{2 \pi \sin ^{2} \theta} 1_{\{0<\theta<\pi / 2\}}
$$

and we consider the following initial distribution of the velocities:

$$
P_{0}(d v)=1_{[-1 / 2,1 / 2]^{3}}(v) d v .
$$

As in Section [5, we define a family of cross sections $\beta_{l}$ (for $l>0$ ) with cutoff by (5.1). We denote by $\left\{P_{t}^{\beta}\right\}_{t}$ and $\left\{P_{t}^{\beta_{l}}\right\}_{t}$ the measure solution of the associated Boltzmann equations, and by $\left\{\mu_{N, t}^{n, l}\right\}_{t}$ (resp. $\left\{\mu_{B, t}^{n, l}\right\}_{t}$ ) the flow of empirical measures associated with the corresponding particle system obtained by the Nanbu (resp. Bird) approach.

Our aim is to "deduce" from simulations how $\mu_{N, t}^{n, l}$ and $\mu_{B, t}^{n, l}$ approach $P_{t}^{\beta}$ in practice. To this aim, we have to consider known quantities. Indeed, we will compare

$$
m_{4}(t)=\int_{\mathbb{R}^{3}}|v|^{4} P_{t}^{\beta}(d v)
$$

with

$$
m_{4}^{l}(t)=\int_{\mathbb{R}^{3}}|v|^{4} P_{t}^{\beta_{l}}(d v)
$$

and with

$$
m_{4, N}^{l, n}(t)=\int_{\mathbb{R}^{3}}|v|^{4} \mu_{N, t}^{n, l}(d v) \quad \text { or } \quad m_{4, B}^{l, n}(t)=\int_{\mathbb{R}^{3}}|v|^{4} \mu_{B, t}^{n, l}(d v) .
$$

First, we will try to study how to choose $n, l$, and the algorithm, in order to obtain the minimal error for a given duration of computation. Then we will study how the error behaves when the time varies. 
7.1. The choice of the algorithm, of $l$ and $n$. In this paragraph, we consider the fixed time $t_{0}=2$. The question we would like to answer is how to choose $l$, $n$, and which algorithm, in order to obtain the best approximation of $m_{4}\left(t_{0}\right)$ by $m_{4, N}^{l, n}\left(t_{0}\right)$ or $m_{4, B}^{l, n}\left(t_{0}\right)$, for a given duration of computation.

First of all, an easy (but fastidious) computation shows that:

$$
\begin{aligned}
& m_{4}\left(t_{0}\right)=\frac{5}{48}\left(1-e^{-\pi t_{0} / 4}\right)+\frac{19}{240} e^{-\pi t_{0} / 4} \\
& m_{4}^{l}\left(t_{0}\right)=\frac{5}{48}\left(1-e^{-(\pi / 4-1 / 2 l) t_{0}}\right)+\frac{19}{240} e^{-(\pi / 4-1 / 2 l) t_{0}} .
\end{aligned}
$$

A first set of simulations shows that the duration of one simulation grows proportionally with respect to the product $n \times l$ and that the algorithm related to Bird's approach is slightly more rapid than that obtained by the Nanbu approach.

We now would like to determine which algorithm looks better. We thus set

$$
\begin{aligned}
\phi_{N}(n, l) & =\frac{100}{m_{4}\left(t_{0}\right)}\left\langle\left|m_{4, N}^{l, n}\left(t_{0}\right)-m_{4}\left(t_{0}\right)\right|\right\rangle, \\
\phi_{B}(n, l) & =\frac{100}{m_{4}\left(t_{0}\right)}\left\langle\left|m_{4, B}^{l, n}\left(t_{0}\right)-m_{4}\left(t_{0}\right)\right|\right\rangle,
\end{aligned}
$$

where \langle\rangle denotes the "mean over many experiences" (we will always take the means over at least 1000 experiences). Thus $\phi_{B}(n, l)$ is the "mean" error, in percent, of one simulation.

A second set of experiences shows that the Bird algorithm looks better. Since it is also slightly more rapid, we use, from now on, the Bird approach.

For a given duration of computation, i.e., for a given $n \times l$, we would like to optimize the approximation. To this aim, we split $\phi_{B}(n, l)$ into $\phi_{B}^{S}(n, l)+\phi^{C}(l)$, where

$$
\phi_{B}^{S}(n, l)=\frac{100}{m_{4}\left(t_{0}\right)} \times\left\langle\left|m_{4, B}^{l, n}\left(t_{0}\right)-m_{4}^{l}\left(t_{0}\right)\right|\right\rangle
$$

is the mean error, in percent, due to one simulation, and

$$
\phi^{C}(l)=\frac{100}{m_{4}\left(t_{0}\right)} \times\left|m_{4}^{l}\left(t_{0}\right)-m_{4}\left(t_{0}\right)\right|
$$

is the "cutoff" error, in percent.

A new set of simulations shows that $\phi_{B}^{S}(n, l)$ depends only very slightly on $l$. Indeed, we see that $\phi_{B}^{S}(n, l)$ satisfies:

\begin{tabular}{|l||l|l|l|l|l|}
\hline$n \backslash l$ & 5 & 10 & 50 & 100 & 200 \\
\hline \hline 10 & 27.90 & 27.96 & 28.41 & 27.66 & 28.01 \\
\hline 100 & 9.11 & 9.20 & 9.32 & 9.37 & 9.39 \\
\hline 1000 & 3.02 & 2.98 & 3.01 & 2.95 & 3.02 \\
\hline
\end{tabular}

Notice that since $\phi^{C}(200) \approx 0.026 \%$, it is not really necessary to consider the case where $l>200$. 
Thus the optimization procedure is quite easy. We first try to determine the behaviour of the function $\phi_{B}^{S}(n) \approx \phi_{B}^{S}(n, l)$. We obtain:

\begin{tabular}{|l||l|l|l|l|l|l|l|l|l|}
\hline$n$ & 100 & 350 & 500 & 1000 & 1500 & 2000 & 2500 & 3000 & 3500 \\
\hline \hline$\phi_{B}^{S}(n, 10)$ & 9.25 & 5.09 & 4.13 & 2.99 & 2.45 & 2.07 & 1.84 & 1.67 & 1.56 \\
\hline$\phi_{B}^{S}(n, 100)$ & 9.79 & 4.98 & 4.34 & 2.92 & 2.39 & 2.09 & 1.90 & 1.66 & 1.60 \\
\hline
\end{tabular}

Thanks to Figure 1 , which describes the previous table, we see that $\phi_{B}^{S}(n) \approx$ $93 / \sqrt{n}$ seems to be a good approximation. This shows, empirically, that $m_{4, B}^{l, n}\left(t_{0}\right)$ satisfies a limit central theorem (in $n$ ), and suggests that a limit central theorem might hold for any "nice" functionnal of $\mu_{t}^{l, n}$. This is not very surprising; see Méléard 11] for a similar problem.

However, since the difference between $93 / \sqrt{n}$ and $\phi_{B}^{S}(n)$ is almost 0 when $n$ varies in $\{100, \ldots, 3500\}$, and since a central limit theorem seems to hold, we might deduce that the approximation $93 / \sqrt{n}$ still holds when $n$ is much larger.

Let us compare briefly this study with that of Desvillettes, Graham, and Méléard, 4], who are interested in the one-dimensional case. In fact, we do not really study the numerical results in the same way as they do. However, one can check, studying Table 1, Section 5 in 4, that (in dimension 1, with the Nanbu system, and with quite the same cross section and initial distribution as (7.1) and (7.2)) one obtains a function $\phi_{N}^{S, 1 D}(n, l) \approx 81 / \sqrt{n}$, for $t_{0}=1.8$. We thus deduce that in practice, the speed of convergence does not depend much on the dimension, even for the constants.

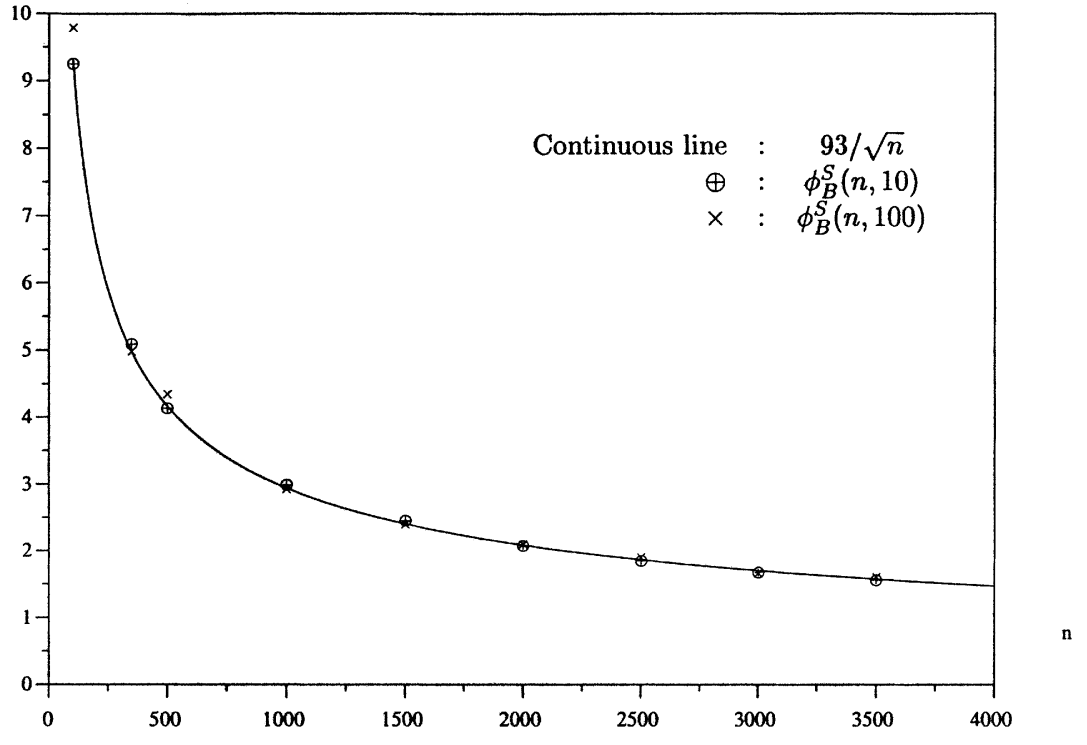

FiguRE 1. Error of one simulation as a function of $n$. 
Let us come back to our problem. A direct computation, using (7.6), shows that

$$
\phi^{C}(l)=5.25 \times\left(e^{1 / l}-1\right) \approx 5.25 / l .
$$

We finally deduce that for a fixed duration of computation $D>>0$, the "best" $n$ and $l$, such that $n \times l=D$, minimizing the total error $\phi_{B}^{S}(n, l)+\phi^{C}(l)$ are given by

$$
n_{D}=4.28 \times D^{2 / 3} ; \quad l_{D}=0.23 \times D^{1 / 3}
$$

which gives a total error behaving as

$$
\phi_{B}^{S}\left(n_{D}, l_{D}\right)+\phi^{C}\left(l_{D}\right)=67.7 \times D^{-1 / 3} .
$$

Of course, the precision given here is probably useless, because the present study holds only for $t_{0}=2$, with the chosen cross section and initial distribution, and only for the moment of order 4 . However, it might give an idea of how to choose $n$ and $l$ in order to obtain quickly a good result in other situations.

7.2. The error as a function of the time. Let us now say a word about the evolution in time of our simulations. We are interested in a comparison between the values of $m_{4}(t)$ and $m_{4, B}^{l, n}(t)$, when $t$ varies. In fact, we would just like to check that the error is not strongly growing when $t$ increases. We obtain Figure 2, choosing $n=3500$ and $l=10$, using only one simulation for each time.

Furthermore, simulating $m_{4, B}^{10,3500}(t)$ once up to $t=500$ (resp. $t=1000$ ), we obtain an error equal to $2.95 \%$ (resp. $1.76 \%$ ), which is equivalent to the errors in Figure 2. We thus "deduce" that our approximations do not become much less and less good when the time increases.

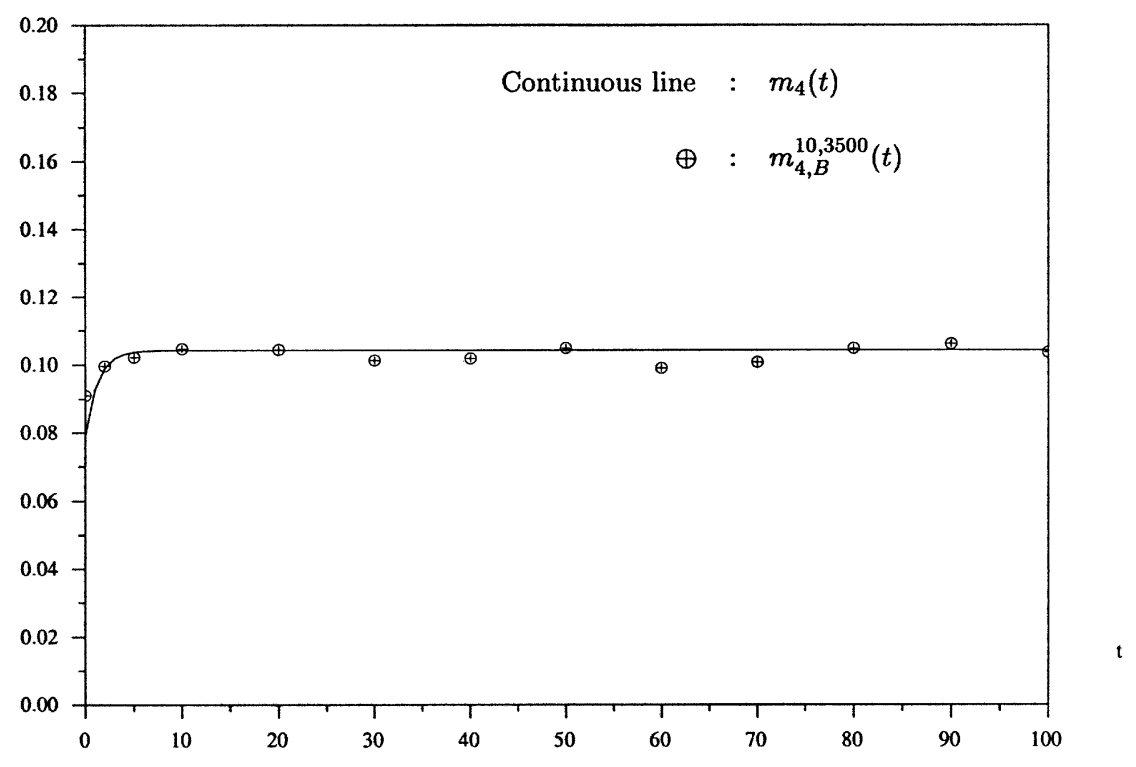

Figure 2. Evolution in time. 


\section{REFERENCES}

[1] Babovsky, H.; Illner, R.: A convergence proof for Nanbu's simulation method for the full Boltzmann equation, SIAM J. Num. Anal. 26 1, 46-65 (1989). MR 90e:76104

[2] Cercignani, C.; Illner, R.; Pulvirenti, M.: The mathematical theory of dilute gases, Applied Math. Sciences, Springer-Verlag, Berlin (1994). MR 96g:82046

[3] Desvillettes, L.: About the regularizing properties of the non-cut-off Kac equation. Comm. Math. Physics 168, 417-440, (1995). MR 96d:82052

[4] Desvillettes, L.; Graham, C.; Méléard, S.: Probabilistic interpretation and numerical approximation of a Kac equation without cutoff, Stoch. Proc. and Appl., 84, 1, 115-135 (1999). MR 2000k: 60127

[5] Fournier, N.: Calcul des variations stochastiques sur l'espace de Poisson, applications à des E.D.P.S. paraboliques avec sauts et à certaines équations de Boltzmann. Thèse de l'Université Paris 6, Chapitre 4, (1999)

[6] Graham, C.; Méléard, S.: Stochastic particle approximations for generalized Boltzmann models and convergence estimates, Ann. Prob.25, 115-132 (1997). MR 98k:60181

[7] Graham, C.; Méléard, S.: Existence and regularity of a solution of a Kac equation without cutoff using the stochastic calculus of variations, Commun. Math. Phys. 205, 551-569 (1999). MR 2001c:60091

[8] Horowitz, J.; Karandikar, R.L.: Martingale problems associated with the Boltzmann equation, Seminar on Stochastic Processes, 1989 (E. Sinlar, K.L. Chung, R.K. GeFoor, eds.), Birkhäuser, Boston (1990). MR 92e:60194

[9] Jacod, J.; Shiryaev, A.N.: Limit theorems for stochastic processes, Springer-Verlag (1987). MR 89k:60044

[10] Méléard, S.: Asymptotic behaviour of some interacting particle systems, McKean-Vlasov and Boltzmann models, cours du CIME 95, Probabilistic models for nonlinear pde's, L.N. in Math. 1627, Springer (1996). MR 98f:60211

[11] Méléard, S.: Convergence of the fluctuations for interacting diffusions with jumps associated with Boltzmann equations, Stochastics and Stoch. Rep., Vol 63, pp 195-225, (1998). MR 99g:60103

[12] Nanbu, K.: Interrelations between various direct simulation methods for solving the Boltzmann equation, J. Phys. Soc. Japan 52, 3382-3388 (1983).

[13] Shiga, T.; Tanaka, H.: Central limit theorem for a system of Markovian particles with meanfield interactions, Z. Wahrsch. Verw. Geb. 69, 439-459 (1985). MR 88a:60056

[14] Sznitman, A.S.: Équations de type de Boltzmann, spatialement homogènes, Z. Wahrsch. verw. Geb. 66, 559-592 (1984). MR 86g:60081

[15] Tanaka, H.: On the uniqueness of Markov process associated with the Boltzmann equation of Maxwellian molecules, Proc. Intern. Symp. SDE, Kyoto, 409-425 (1976). MR 81d:60063

[16] Tanaka, H.: Probabilistic treatment of the Boltzmann equation of Maxwellian molecules, Z. Wahrsch. Verw. Geb. 46, 67-105 (1978). MR 80b:60083

[17] Toscani, G.; Villani, C.: Probability metrics and uniqueness of the solution to the Boltzmann equation for a Maxwell gas, J. Stat. Phys., 619-637 (1999). MR 2000f:82089

[18] Wagner, W.: A convergence proof for Bird's direct simulation method for the Boltzmann equation, J. Stat. Phys. 66, 1011-1044 (1992). MR 93f:82053

Institut Elie Cartan, Faculté de Sciences, B.P. 239, 54506 Vandoeune-Les-Nancy Cedex, France

E-mail address: Nicolas.Fournier@antares.iecn.u-nancy.fr

Laboratoire de Probabilités, Paris 6, 4 Place Jussieu, 75252 Paris Cedex 05, France; and MODAlX, UFR SEGMi, Université Paris 10, 92000 Nanterre, France

E-mail address: sylm@ccr.jussieu.fr 